\title{
Understanding the impact of Light cone effect on the EoR/CD 21-cm power spectrum
}

\author{
Kanan K. Datta ${ }^{1}$, Rajesh Mondal ${ }^{2,3}$, Raghunath Ghara ${ }^{4}$, Somnath \\ Bharadwaj $^{2}$ and T. Roy Choudhury ${ }^{5}$ \\ ${ }^{1}$ Physics Department, Presidency University, 86/1 College Street, Kolkata-73, WB, India \\ ${ }^{2}$ Centre for Theoretical Studies, Indian Institute of Technology Kharagpur-721302, India \\ ${ }^{3}$ Astronomy Centre, Department of Physics and Astronomy, University of Sussex, Pevensey II \\ Building, Falmer, Brighton BN1 9QH \\ ${ }^{4}$ Department of Astronomy and Oskar Klein Centre, AlbaNova, Stockholm University, SE-106 \\ 91 Stockholm, Sweden \\ ${ }^{5}$ National Centre for Radio Astrophysics, Tata Institute of Fundamental Research,Post Bag 3, \\ Ganeshkhind, Pune 411 007, India \\ email: kanan.physics@presiuniv.ac.in
}

\begin{abstract}
Redshifted HI 21-cm signal from the cosmic dawn and epoch of reionization evolve considerably along the LoS. We study the impact of this evolution (so called the light cone effect) on the HI 21-cm power spectrum. It is found that the LC effect has a significant impact on the 3D power spectrum and the change could be up to a factor of few. The LC effect is particularly strong during the cosmic dawn near the 'peaks' and 'dips' in the power spectrum when plotted with redshift. We also show that the 3D power spectrum, which could fully describe ergodic and periodic signal, losses out some information regarding the second order statistics of the signal as the EoR/CD 21-cm signal is non-ergodic and non-periodic along the line of sight. We show that the multi-frequency angular power spectrum (MAPS) $\mathcal{C}_{\ell}\left(\nu_{1}, \nu_{2}\right)$ captures all the information regarding the second order statistics of the signal even in the presence of the LC effect.
\end{abstract}

Keywords. Cosmology: Epoch of Reionization, Cosmic Dawn, 21-cm line, Theory \& Simulations, Statistical analysis

\section{Introduction}

Observations of redshifted 21-cm radiation from neutral hydrogen (HI) from the epoch of reionization (EoR) and cosmic dawn (CD) are considered to be one of the most promising tools for probing the epoch and currently the frontier of modern cosmology. The power spectrum of HI differential brightness temperature fluctuations during EoR and CD contains enormous amount of information regarding the nature of first sources, timing and duration of the event. It is the primary target of the first generation and upcoming lowfrequency radio telescopes. However, the average signal as well as the statistical properties of HI 21-cm signal evolve with redshift. This effect, known as 'light-cone' (LC) effect, has a considerable impact on the HI 21-cm power spectrum. It is thus important to asses the effects of the LC effect on the HI 21-cm signal and its power spectrum in order to understand its role on the statistics of the signal and interpretation of observed signal.

Datta et al. (2012) use large scale numerical simulations and investigate the impact of the LC effect on the spherically averaged HI power spectrum. It finds that the effect mainly 'averages out' and reports a change of up to $\sim 50 \%$ at the large scales corresponding to a frequency bandwidth of $\sim 8 \mathrm{MHz}$. Subsequently, $\sim \mathrm{Gpc}$ size simulations have been used to investigate various other issues such as quantifying the LC induced anisotropies in the power spectrum and determining the optimal bandwidth for analyzing the observed 


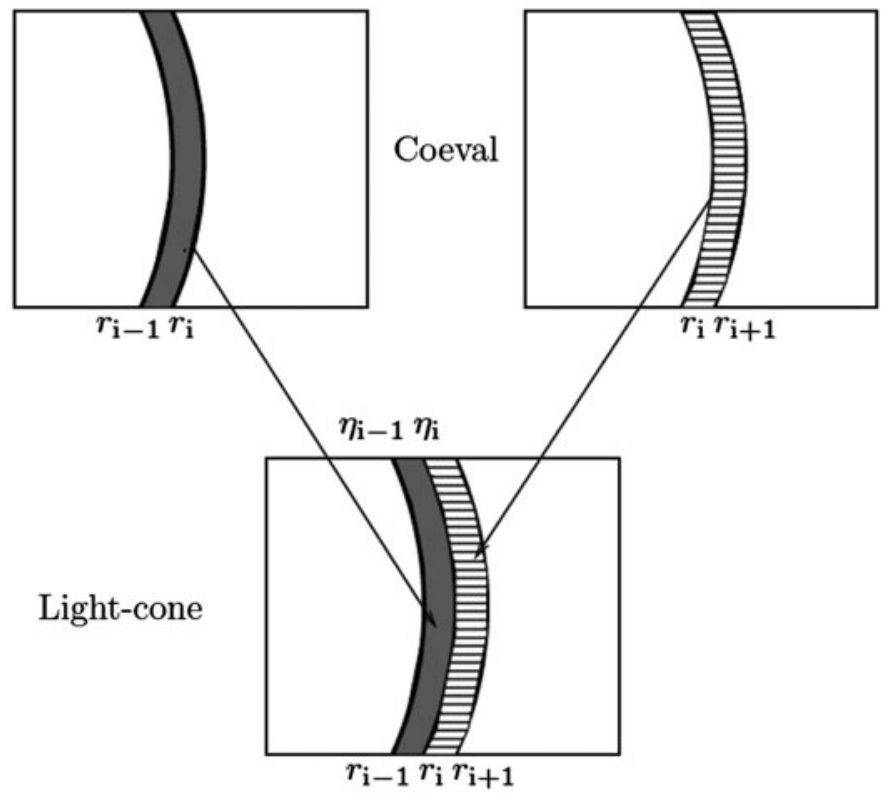

Figure 1. Schematic diagram showing how we sliced the coevalsimulations and stitched the slices to form the LC simulation (taken from Mondal et al. (2018).

signal (La Plante et al. 2014; Datta et al. 2014). Ghara et al. (2015) further extend the work and consider the HI 21-cm signal from the cosmic dawn which includes fluctuations in the spin temperature. It finds that the LC effect has a dramatic signature on the cosmic dawn HI power spectrum. Recently, Greig \& Mesinger (2018) investigate the impact of LC effect on the estimation of EoR source parameters. Issues such as how to properly include the LC effect in the presence of peculiar velocity and analyze the statistics of the light cone EoR/CD 21-cm signal have been studied in Mondal et al.(2018).

This paper presents a summary of recent works on LC effects on EoR/CD 21-cm signal and address issues which might be important while simulating LC 21-cm signal and quantifying statistically through power spectrum. At the end, we introduce the multifrequency angular power spectrum (MAPS) and argue that it is better suited to quantify statistics of EoR/CD 21-cm signal compared to 3D power spectrum as it does not assume the signal to be periodic and ergodic along the line of sight (LoS) and captures the full information of the HI 21-cm signal.

\section{Simulating light cone $21-\mathrm{cm}$ signal}

The EoR and CD simulations provide us 3D cubes of HI density and HI fraction at the same redshift i.e, so-called coeval cubes. However, an observer cannot observe these coeval cubes as different parts along the line of sight of an observed volume correspond to different redshifts or cosmic time. Therefore, we use coeval cubes simulated at different redshifts covering the entire $\mathrm{CD} / \mathrm{EoR}$ to simulate light-cone boxes. Two different methods have been introduced to simulate LC boxes from coeval cubes: one can be implemented on gridded data and thus faster (see Datta et al. (2012) for details), the other one is a particle based method which requires particle positions and velocities. The second method is more accurate but requires more computing time. Here, we briefly discuss the second method which is implemented in Mondal et al.(2017). 
The method first simulates coeval cubes at $N$ different epochs $\eta_{i}$ ( $\eta$ being the conformal time) at non-uniform intervals of $\Delta \eta_{i}$ which are chosen so that the neutral fraction $\bar{x}_{\mathrm{HI}}$ varies by approximately an equal amount in each interval†. The HI distribution in simulations consists of particles whose HI masses vary with position depending on the local HI fraction. Each coeval simulation provides particle positions, velocities (peculiar) and HI masses. To construct the LC simulation we slice the simulation volume at comoving distances $r_{i}$ corresponding to conformal time $\eta_{i}$ at which coeval cubes have been generated. For each slice we fill in the region $r_{i}$ to $r_{i+1}$ with the particles from the corresponding region in the coeval cubes at the epoch $\eta_{i}$ (Fig. 1).

Observations will provide differential brightness temperature fluctuations $\delta T_{b}(\hat{n}, \nu)$ where $\nu$ is the observing frequency and $\hat{n}$ is the unit vector in the direction of observation. Thus, we map simulated light cone boxes to $(\hat{n}, \nu)$ coordinates. The observing frequency depends both on the cosmological redshift (arising solely due to Hubble velocity) and the peculiar velocity. We consider the effects of peculiar velocity while mapping particle positions obtained from simulation to $(\hat{n}, \nu)$ coordinates. Simulation boxes are then gridded in $(\hat{n}, \nu)$ space with uniform grid in solid angle $(\Delta \Omega)$ and frequency $(\Delta \nu)$ and HI density is calculated at each $(\hat{n}, \nu)$ grid. Finally, the differential brightness temperature fluctuations $\delta T_{b}$ is calculated in comoving coordinates by reverse mapping (see Mondal et al.(2017) for details).

\subsection{Simulating coeval cubes}

Both simulations first obtain the dark matter density distribution and halo catalogue from two separate N-body codes. The first simulation uses an $1 \mathrm{D}$ radiative transfer algorithm to generate differential brightness temperature fluctuations $\delta T_{b}$ maps at different redshifts covering the entire cosmic dawn and the EoR. It also considers the fluctuations in the spin temperature by incorporating inhomogeneous X-ray heating and Ly- $\alpha$ coupling during the cosmic dawn. Then the first grid based method mentioned above is used while generating the LC boxes. More details regarding simulation details and sources model can be found in Ghara et al. (2015a, 2015). The second simulation uses a semi-numerical technique (Choudhury et al. (2009), Majumdar et al. (2013)) to simulate the coeval ionization cubes with a comoving length $L=300.16 \mathrm{Mpc}$ on each side. As described above, the HI distribution in these simulations is represented by particles whose HI masses were calculated from the neutral Hydrogen fraction $\bar{x}_{\mathrm{HI}}$. Each co-eval box provides the HI masses, positions, peculiar velocities of these particles. It uses the particle based method described above to generate LC simulations.

\section{Effects on spherically averaged power spectrum}

Fig. 2 shows the LC effect on the spherically averaged power spectrum of the $21-\mathrm{cm} \delta T_{b}$ fluctuations. Left panel shows results for the first simulation (model C, see Ghara et al. (2015) for deatils) which considers spin temperature fluctuations arising due to inhomogeneous X-ray heating and Ly- $\alpha$ coupling during cosmic dawn. It shows the evolution of the dimensionless power spectrum as a function of redshift for four different scales $k=0.05,0.1,0.5$ and $1 \mathrm{Mpc}^{-1}$ which are represented by the solid, dotted, dashed and dot-dashed curves, respectively. We see that the evolution of the power spectrum is quite dramatic with several ups and downs when plotted as a function of redshift. There are three peaks at redshifts $z \sim 17,14$ and 8 in the power spectrum and the amplitude goes to as high as $\sim 200 \mathrm{mK}^{2}$ and comes down to $\sim 0.5 \mathrm{mK}^{2}$ at later redshifts for $k \sim 0.05 \mathrm{Mpc}^{-1}$.

$\dagger$ Note that, coeval cubes, in the first method, are generated at equal cosmic time interval $\delta t$ 

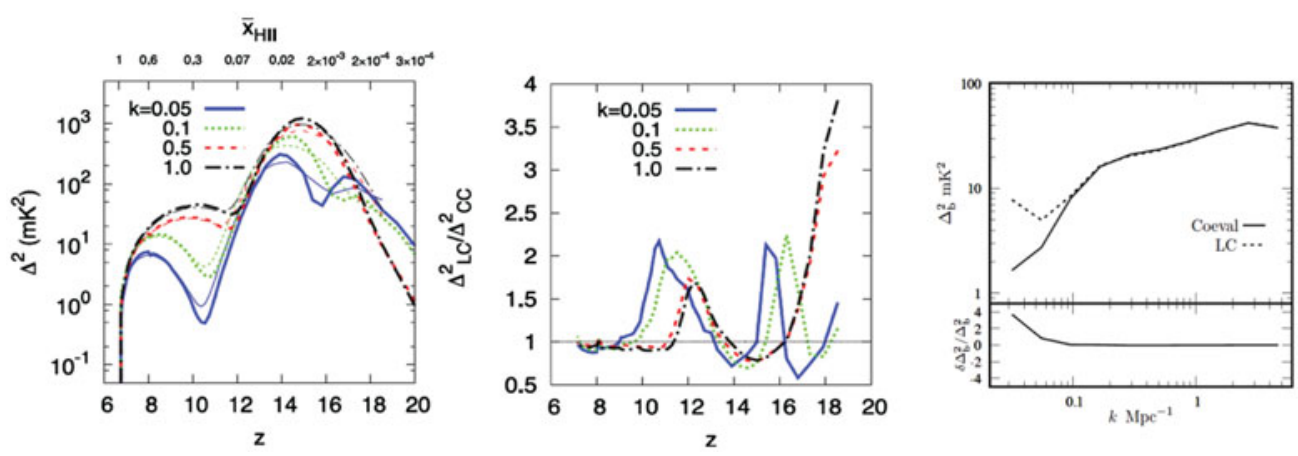

Figure 2. The LC effect on the power spectrum of 21-cm brightness temperature fluctuation. The left panel shows the spherically averagedpower spectrum with (thin curves) and without (thick curves) LC effect. The middle panel shows the ratio of the power spectra with and without LC effect. The left and the middle panels are taken from Fig. 6 of Ghara et al. (2015) and corresponds to model $\mathrm{C}$ where both Ly- $\alpha$ coupling and heating are calculated self-consistently. The right panel, which is taken from Mondal et al. (2018), show the spherically averagedpower spectrum with (solid line) and without (dashed line) LC effect. The bottom panel of the right shows the ratio. The grid and particle based methods are used to generate LC boxes for the left and right panels respectively.

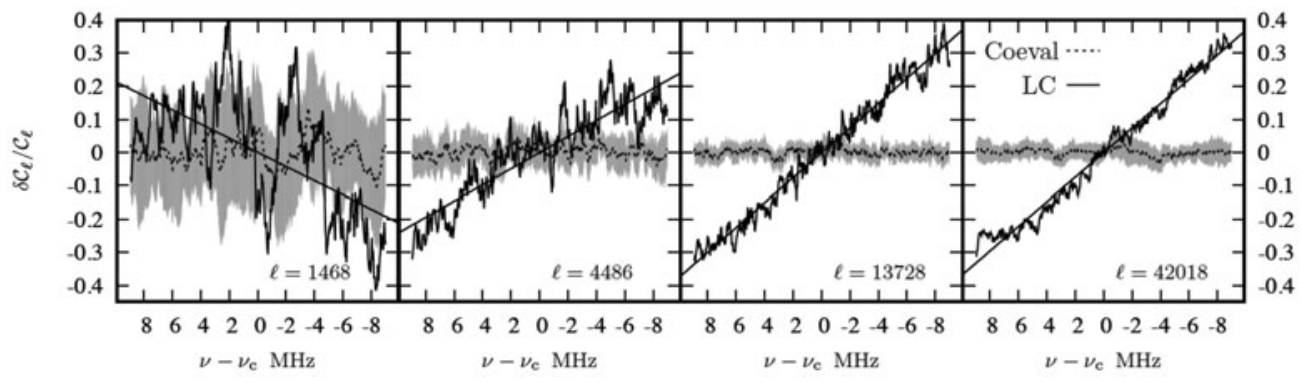

Figure 3. The dimensionless $\delta \mathcal{C}_{\ell} / \mathcal{C}_{\ell}$ for the $l$ values shown in the figure. We also show straight line fits to $\delta \mathcal{C}_{\ell} / \mathcal{C}_{\ell}$ estimated from the LCsimulation. We have used 10 statistically independent realizations of the coeval simulation to estimate the mean $\delta \mathcal{C}_{\ell} / \mathcal{C}_{\ell}$ which is close tozero, and the $1-\sigma$ fluctuations which have been shown by the shaded regions. The figure is taken from Mondal et al. (2018).

We also find maximum suppression of power spectrum occurs around the two peaks and enhancement around the trough region between the two peaks. In presence of inhomogeneous X-ray heating and Ly- $\alpha$ coupling the evolution of the power spectrum is more dramatic at both large and small scales at cosmic dawn. The non-linear rise of the power spectrum due to inhomogeneous X-ray heating and Ly- $\alpha$ coupling, results in a stronger LC effect at the very beginning of the reionization epoch.

The right panel of Fig. 2 shows HI power spectrum for the second simulation with and without the LC effect included. Although, simulations are quite different and uses two different methods to implement the LC effect the results are qualitatively similar i.e, the LC effect has significant impact at large scales.

\section{Towards quantifying light cone 21 -cm signal}

The EoR/CD HI 21-cm signal evolves considerably along the LoS direction $\nu$ due to the LC effect (Fig. 2). It is important to note that the 3D Fourier modes and 3D power 
spectrum $P(k)$ are optimal if the signal is statistically homogeneous. The 3D Fourier modes are not the optimal basis set when the statistical properties of the signal evolve along one axis. Additionally, the Fourier transform imposes periodicity on the signal, an assumption that is not true along the LoS once the LC effect is included. Consequently, the 3D power spectrum fails to fully quantify the entire signal. We introduce the multifrequency angular power spectrum $\mathcal{C}_{\ell}\left(\nu_{1}, \nu_{2}\right)$ (hereafter MAPS) to address this issue

$$
C_{l}\left(\nu_{1}, \nu_{2}\right)=\left\langle a_{l m}\left(\nu_{1}\right) a_{l m}^{*}\left(\nu_{2}\right)\right\rangle
$$

This incorporates the assumption that the EoR 21-cm signal is statistically homogeneous and isotropic with respect to different directions in the sky, however the signal is not assumed to be statistically homogeneous along the LoS direction $\nu$. Thus the MAPS $\mathcal{C}_{\ell}\left(\nu_{1}, \nu_{2}\right)$ quantifies the entire second orderstatistics of the EoR 21-cm signal even in the presence of the LC effect. In the flat sky approximation, one can show that $P(\mathbf{k})$ can be calculated using (Datta et al. 2007)

$$
P\left(k_{\perp}, k_{\|}\right)=r_{\mathrm{c}}^{2} r_{\mathrm{c}}^{\prime} \int d(\Delta \nu) e^{-i k_{\|} r_{\mathrm{c}}^{\prime} \Delta \nu} \mathcal{C}_{\ell}^{\mathrm{EP}}(\Delta \nu)
$$

where $k_{\|}$and $k_{\perp}=\ell / r_{\mathrm{c}}$ are the components of $\mathbf{k}$ parallel and perpendicular to the LoS respectively. Here $\mathcal{C}_{\ell}^{\mathrm{EP}}(\Delta \nu)$ is the MAPS assuming the signal to be periodic $(\mathrm{P})$ and ergodic (E). A brief derivation of eq. (4.2) is presented in the Appendix A of Mondal et al. (2018). This implies that $P(\mathbf{k})$ can be fully recovered from MAPS if the signal is assumed to be periodic and ergodic. However, $\mathcal{C}_{\ell}\left(\nu_{1}, \nu_{2}\right)$ is more generic and contains the entire second order statistics of the EoR 21-cm signal even in the presence of the LC effect. The difference $\delta \mathcal{C}_{\ell}\left(\nu_{1}, \nu_{2}\right)=\mathcal{C}_{\ell}\left(\nu_{1}, \nu_{2}\right)-\mathcal{C}_{\ell}^{\mathrm{EP}}\left(\nu_{1}, \nu_{2}\right)$ gives an estimate of the information that is missing in the $3 \mathrm{D}$ power spectrum $P(\mathbf{k})$. We focus on the diagonal terms $\nu_{1}=\nu_{2}$ where the MAPS signal peaks (Fig. 6 of Mondal et al. (2018)). Fig. 3 shows how $\delta \mathcal{C}_{\ell} / \mathcal{C}_{\ell}^{\text {EP }}$ varies with $\nu$ for four different values of $\ell$. We note that the denominator does not vary with $\nu_{1}$ for the diagonal terms. We see that $\delta \mathcal{C}_{\ell} / \mathcal{C}_{\ell}$ estimated from the coeval simulations shows random fluctuations around zero, and is consistent with zero. These random fluctuations as arising due to cosmic variance. In contrast, we find that $\delta \mathcal{C}_{\ell} / \mathcal{C}_{\ell}^{\mathrm{EP}}$ shows a systematic variation with $\nu_{\mathrm{i}}$ in the $\mathrm{LC}$ simulation. This variation is particularly noticable at large $\ell$ where the value of $\delta \mathcal{C}_{\ell} / \mathcal{C}_{\ell}^{\text {EP }}$ varies systematically from $\sim-0.4$ to $\sim 0.4$ with decreasing frequency. This clearly indicates that the $3 \mathrm{D}$ power spectrum loses out $\sim 40 \%$ of the information at the two ends of our band.

\section{Summary \& Conclusions}

The global 21-cm signal and its statistics evolve substantially with redshift during the EoR. The evolution is quite dramatic during the cosmic dawn. This paper summarises works which study the impact of this so called light cone effect on the HI power spectrum which is the primary target of all ongoing and upcoming EoR 21-cm experiments. It is found that the LC effect has a significant effect on the 3D HI 21-cm power spectrum and the change could be up to a factor of few depending on the observational bandwidth and stages of EoR/CD. The effect is particularly strong when the underlying 3D coeval power spectrum evolve non-linearly. It also smooths out/enhances the peaks/troughs in the $3 \mathrm{D}$ power spectrum which is particularly noticeable during cosmic dawn.

We, then, critically investigate whether the 3D power spectrum captures the full information encoded in the LC EOR/CD 21-cm signal. The 3D Fourier modes and 3D power spectrum $P(k)$ are optimal if the signal is statistically homogeneous. The 3D Fourier modes which are used to calculate $P(k)$ are not the optimal basis set when the statistical 
properties of the signal evolve within the simulation volume. Additionally, the Fourier transform imposes periodicity on the signal, an assumption that is not true along the LoS once the LC effect is included. This means that the 3D and spherically averaged HI 21-cm power spectrum is not able to fully describe the signal and loses out some information. We argue that the multi-frequency angular power spectrum (MAPS) which is calculated using 2D Fourier modes of signal in the sky plane captures the entire second order statistics of the EoR 21-cm signal even in the presence of the LC effect. It can also be shown that under the assumption of homogeneous and period signal along the line of sight axis the MAPS can be equivalent to 3D power spectrum.

\section{References}

Choudhury, T. R., Haehnelt, M. G., \& Regan, J, 2009, MNRAS, 394, pp. 960

Datta K. K., Choudhury T. R., \& Bharadwaj S., 2007, MNRAS, 378, 119

Datta K. K., Mellema G., Mao Y., Iliev I. T., Shapiro P. R., \& Ahn K. 2012, MNRAS, 424, 1877

Datta K. K., Jensen H., Majumdar S., Mellema G., Iliev I. T., Mao Y., Shapiro P. R., \& Ahn K. 2014, MNRAS, 442, 1491

Ghara R., Choudhury T. R., \& Datta K. K. 2015a, MNRAS, 447, 1806G

Ghara R., Datta K. K., \& Choudhury T. R. 2015, MNRAS, 453, 3143

Greig B. \& Mesinger A 2018, arxiv: 1801.01592

La Plante P., Battaglia N., Natarajan A., Peterson J. B., Trac H., Cen R., \& Loeb A. 2014, APJ, 789, 31

Majumdar, S, Bharadwaj, S, \& Choudhury, T. R., 2013, 434, p.1978

Mondal R., Bharadwaj S., \& Datta K K., 2018, MNRAS, 474, 1390 